\title{
On Simulation of the Deterministic Model of Drug Infusion and Dissemination in Human Beings
}

\author{
${ }^{1}$ Obayomi, A.A., ${ }^{2}$ Oke, M.O., ${ }^{3}$ Salaudeen, L.O. and ${ }^{4}$ Adetolaju, O.S \\ ${ }^{1,2}$ Department of Mathematics, Ekiti State University, Ado - Ekiti, Nigeria \\ ${ }^{3}$ Department of Mathematics, Federal University Oye, Ekiti State, Nigeria \\ ${ }^{4}$ Department of Computer Sciences, Ekiti State University, Ado - Ekiti, Nigeria
}

\begin{abstract}
In this paper, we presented a new scheme which is a combination of Standard and nonstandard finite difference method. The scheme is a one step method which can be used to solve ordinary differential equations that arises from the dynamics of drug infusion and dissemination in human beings. We applied the scheme to the solubility component of the deterministic model of drug diffusion. The numerical experiment has shown that the scheme can be used for the simulation of drug dissemination in human beings.
\end{abstract}

Keywords: Drug Dissemination, Interpolating function, Nonstandard schemes, Solubility, Drug Infusion, Deterministic Model, Dynamic system

\section{Introduction}

Dynamical systems can be classified as discrete time or continuous time systems depending on the mode of occurrence. If it occurs in such a way that it involves every time slice of the study or prescription then it is said to be continuous. If the dynamic actions occur at segmented points during the time slice under consideration or prescription then it is said to be discrete. The desire for solution of dynamic systems is to be able to track the behavior of the system during a particular period of time.

\section{Drug Dissemination}

Dissolution process refers to the mixing through attraction and association of molecules of a solvent with molecules of a solute. Drug infusion or dissemination is a form of dissolution where active ingredients of a drug are introduced into the blood system to generate certain reaction that produces some sort of relief to human beings. Even though the attraction and association with certain elements of the body system is complex, the concentration function can be viewed as that of decay system for the drug components and a growth system or increment in state of the affected components of the body system.

The Noyes-Whitney equation provides much practical information relevant to the dissolution process. It predicts that the diffusion coefficient which is in part related to solvent viscosity will decrease with increasing solvent viscosity, and decreasing dissolution rate. Thus the diffusion coefficient is inversely proportional to viscosity, Remington Education Publications (2016).

It also predicts that dissolution rate will be faster with smaller particles because surface area increases as particle size decreases. Therefore, micronization of particles will usually speed up dissolution, Mohammed and Hayat (2005) and Remington Education Publications (2016). The Noyes-Whitney equation also shows that some agitation during dissolution will decrease the diffusion gradient by removing solute molecules more quickly from the particle surface, Mohammed and Hayat (2005) and Remington Education Publications (2016). The mathematical models of dissolution are commonly grouped into two types: Deterministic and Stochastic. These two types actually investigate the whole population of particles and describe the time course of concentration $f(t)$. The major difference is that the deterministic models work with given function $f(t)$ while the stochastic models assumes a random process for the dissolution. The concentration function is generally assumed to be continuous and smooth (with derivatives of all orders). $f(t)$ is a non-decreasing function of time. For now, we will consider a simple basic deterministic model which can be represented by differential equation of the form

$y^{\prime}=r-k y$

where $r$ and $k$ are positive constants, $y(t)$ is the concentration of the drug in the blood stream at time $t$ in line with the works of Noyes and Whitney, Remington Education Publications (2016).

This first order differential equation has a solution of the form

$y(t)=y_{0}\left(1-e^{-a t}\right)$

Without loss of generality, we will assume an interpolation function of the form 
$y(x)=a_{0}+a_{1} e^{-\alpha x}$

Equation (3) is therefore used for simulating the dynamics of drug dissolution in human.

The objective of this paper is to develop a new numerical model that correctly replicates the behavioral pattern and dynamics of drug dissemination. Mickens (1992), Angueluv and Labuma (2000), Mickens (1994), and Angueluv and Labuma (2003) have laid a standard foundation for modeling using nonstandard methods to create numerical schemes that possess some stability properties. This work has been extended by many researchers which include Obayomi and Oke (2015), Ibijola et al. (2013), Obayomi and Olabode (2013), Obayomi and Oke (2016) and Obayomi et al. (2017) In this research work, we will particularly create a discrete model that can be used to monitor drug dissemination in mammals using a single drug component to be applied once where the initial conditions are known and approved. The work will combine the techniques of nonstandard and standard method to create a new numerical scheme that can be used to analyze the solution of dynamic systems whose behavior can be represented as an interpolating equation given in (3) above.

From equation (3), we have

\section{Derivation of the Schemes}

$a_{0}=y(x)-a_{1} e^{-\alpha x}$

$y^{\prime}(x)=-\alpha a_{1} e^{-\alpha x}$

$a_{1}=\frac{-y v(x)}{\alpha e^{-\alpha x}}$

Putting (6) in (4), we have

$a_{0}=y(x)-e^{\alpha x} \frac{y^{y}(x)}{\alpha}$

But

$y\left(x_{n}\right)=a_{o}+a_{1} e^{-\alpha x_{n}}$

$y\left(x_{n+1}\right)=a_{0}+a_{1} e-{ }^{\alpha x_{n+1}}$

$y\left(x_{n+1}\right)-y\left(x_{n}\right)=+a_{1} e-\alpha x_{n+1}-a_{1} e^{-\alpha x_{n}}$

$y\left(x_{n+1}\right)=y\left(x_{n}\right)+a_{1}\left(e^{-\alpha x_{n+1}}-e^{-\alpha x_{n}}\right)$

Putting (6) in (8), we have

$y\left(x_{n+1}\right)=y\left(x_{n}\right)-\frac{y^{f(x)}}{\alpha e^{-\alpha x}}\left(e^{-\alpha x_{n+1}}-e^{-\alpha x_{n}}\right)$

Suppose

$x_{n}=a+n h$

$x_{n+1}=a+(n+h)$

Then, (9) becomes:

$y\left(x_{n+1}\right)=y\left(x_{n}\right)-\frac{y^{\prime}(\alpha)}{\alpha e^{-\alpha(a+n h)}}\left(e^{-\alpha(\alpha+(n+1) h}-e^{-\alpha(a+n h)}\right)$

which can be written as

$y_{n+1}=y_{n}-\frac{f n}{\alpha e^{-\alpha(a+n h)}}\left(e^{-\alpha(\alpha+(n+1) h}-e^{-\alpha(\alpha+n h)}\right)$

Equation (10) is the required standard finite difference scheme.

This will be renormalized by applying rule 2 of the nonstandard modeling rules. We will therefore obtain two new schemes by replacing $\mathrm{h}$ with a dynamic function of $\mathrm{h}$ as follows

$\psi(h) \rightarrow h+0\left(h^{2}\right)$ as $h \rightarrow 0$.

$\psi=\sin (h), \quad \psi=\frac{\left(e^{\lambda n}-1\right)}{\lambda}, \psi=\sin (\propto h), \psi=h \quad \propto, \lambda \in \mathbb{R}$

\section{Proof of Convergence}

The incremental function for equation (10) can be written as

$$
\begin{aligned}
& \phi\left(x_{n}, y_{n}, h\right)=-\frac{f n}{\alpha e^{-\alpha(a+n h)}}\left(e^{-\alpha(\alpha+(n+1) h}-e^{-\alpha(\alpha+n h)}\right) \\
& \phi\left(x_{n}, y_{n}, h\right)=A f_{n} \\
& \phi\left(x_{n}, y_{n}, h\right)-\phi\left(x_{n}, y_{n}^{*}, h\right)=A\left[f\left(x_{n}, y_{n}, h\right)-f\left(x_{n}, y_{n}^{*}, h\right)\right] \\
& =A\left[f\left(x_{n}, y_{n}\right)-f\left(x_{n}, y_{n}^{*}\right)\right]
\end{aligned}
$$


$=A\left[\frac{\partial f\left(x_{n n} \bar{y}\right)}{\partial y_{n}}\left(y_{n}-y_{n}^{*}\right)\right]$

$\mathrm{L}=\operatorname{SUP}_{\left(x_{n} y_{n}\right) \in D} \frac{\partial f\left(x_{n} \bar{y}\right)}{\partial y_{n}}$ and

then

$\phi\left(x_{n}, y_{n}, h\right)-\phi\left(x_{n,} y_{n}^{*}, h\right)=A\left[L\left(y_{n}-y_{n}^{*}\right)\right]$

Let $\mathrm{M}=|\mathrm{A} . \mathrm{L}|$

$\phi\left(x_{n}, y_{n}, h\right)-\phi\left(x_{n}, y_{n}^{*}, h\right) \leq M\left|y_{n}-y_{n}^{*}\right|$

Equation (13) indicates the condition for convergence.

V. Consistency of the schemes

$y_{n+1}=y_{n}-\frac{f n}{\alpha e^{-\alpha\lfloor a+n h)}}\left(e^{-\alpha(\alpha+(n+1) h}-e^{-\alpha(\alpha+n h)}\right)$

$y_{n+1}=y_{n}+\{A\} f_{n}$

When $h=0, A=0$

$\Rightarrow y_{n+1}=y_{n}$ and the incremental function is identically zero when $h=0$

$\Rightarrow \phi\left(x_{n}, y_{n}, 0\right) \equiv 0$

Consider the equation

\section{Stability of the schemes}

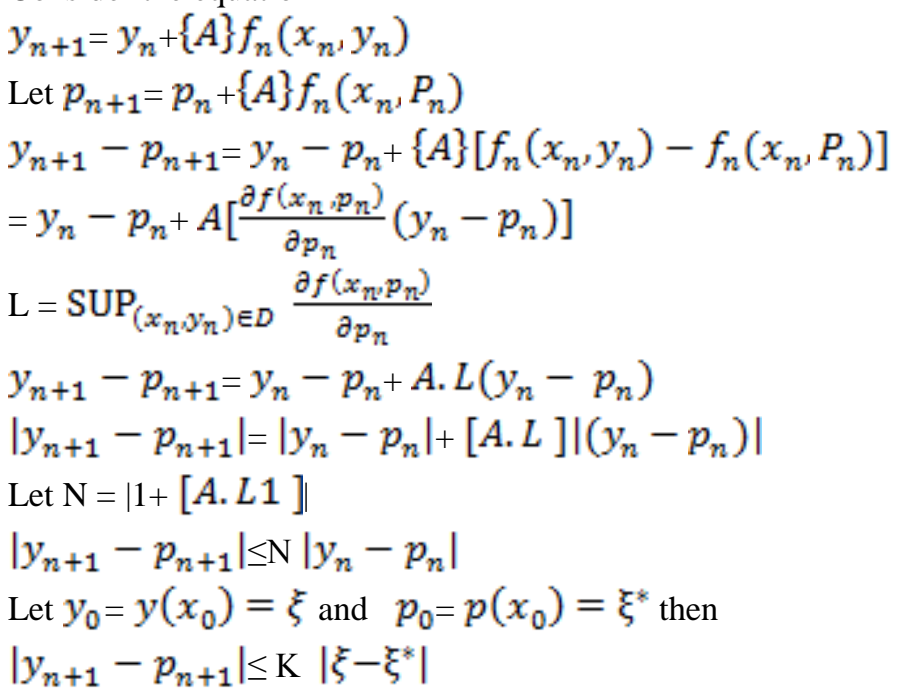

Problem 1

\section{Numerical Experiment}

Let us consider the initial value problem below from Zill and Cullen (2005)

$$
\begin{aligned}
y^{\prime} & =4 x-2 x y, y(0)=3 \\
f_{n} & =y_{\left(x_{n}\right)}^{\prime}=4 x_{n}-2 x_{n} y_{n} \\
f_{n}^{\prime} & =y^{\prime \prime}\left(x_{n}\right)=4-2 y_{n}^{\prime}-2 y_{n} \\
f_{n}^{\prime} & =y^{\prime \prime}\left(x_{n}\right)=4-8 x_{n}^{2}+4 x_{n}^{2} y_{n}-2 y_{n}
\end{aligned}
$$

Substituting for $f n$ in equation (10), we have

$y_{n+1}=y_{n}-\frac{4 x_{n}-2 x_{n} y_{n}}{\alpha e^{-\alpha(a+n h)}}\left(e^{-\alpha(\alpha+(n+1) h}-e^{-\alpha(\alpha+n h)}\right)$

Three hybrid schemes will be obtained by replacing $h$ by $\psi=\sin (h)$ and $\psi=\frac{\left(e^{\lambda n}-1\right)}{\lambda}$ in equation (15) to obtain NewSin for $\psi=\sin (h)$, New Exp when $\psi=\frac{\left(e^{\lambda h}-1\right)}{\lambda}$ and Newh for the scheme with $\mathrm{h}$ not normalized. 
The analytic solution is $y=2 e^{x}-x-1$

The Nonstandard scheme using rules 2 and 3 will be

$\frac{\left(\mathrm{y}_{\mathrm{n}+1}-\mathrm{y}_{\mathrm{n}}\right)}{\Psi}=4 x_{n}-2 x_{n}\left(c y_{n+1}+d y_{n}\right)$

$y_{n+1}=y_{n}+\frac{y_{n}(1-2 \psi d)+4 \psi x}{(1+2 x \psi c)}$

Problem 2

Let us consider the initial value problem below from Zill and Cullen (2005)

$$
\begin{gathered}
y^{\prime}=y^{2}-4, y\left(\frac{1}{4}\right)=1 \\
f_{n}=y_{\left(x_{n}\right)}^{\prime}=y_{n}^{2}-4
\end{gathered}
$$

Substituting for $f n$ in equation (10), we have

$y_{n+1}=y_{n}-\frac{y_{n}^{2}-4}{\alpha e^{-\alpha(a+n h)}}\left(e^{-\alpha(\alpha+(n+1) h}-e^{-\alpha(\alpha+n h)}\right)$

Again, three hybrid schemes will be obtained by changing $\mathrm{h}$ to $\psi=\sin (h)$ and $\psi=\frac{\left(e^{\lambda \mathrm{n}}-1\right)}{\lambda}$ in equation (19).

The analytic solution is $y=2\left[\frac{3-e^{4 x-1}}{3+e^{4 x-1}}\right]$

The Nonstandard scheme using rules 2 and 3 will be

$\frac{\left(\mathrm{y}_{\mathrm{n}+1}-\mathrm{y}_{\mathrm{n}}\right)}{\psi}=\left(c y_{n} y_{n+1}+d y_{n}^{2}\right)-4$

$y_{n+1}=\frac{y_{n}\left(1+\psi d y_{n}\right)-4 \psi}{\left(1+c \psi y_{n}\right)}$

\section{Drug Dissemination Model}

Applying the standard finite difference scheme in equation (10) to the drug dissolution equation $y^{\prime}=r-k y=f n$, we have

$y_{n+1}=y_{n}-\frac{r-k y_{n}}{\alpha e^{-\alpha(a+n h)}}\left(e^{-\alpha(\alpha+(n+1) h}-e^{-\alpha(a+n h)}\right)$

The Nonstandard scheme using rules 2 and 3 will be

$$
y^{\prime}=r-k y
$$

$\frac{\left(\mathrm{y}_{\mathrm{n}+1}-\mathrm{y}_{\mathrm{n}}\right)}{\Psi}=r-c k y_{n+1}-d k y_{n}$

$y_{n+1}=\frac{y_{n}(1-k \psi d)+\psi r}{(1+k \psi c)}$

\section{Results of Numerical Experiment}

The algorithm of these schemes has been coded into a software program and it has been tested using several step sizes. The sample result for $h=0.01$ is given below

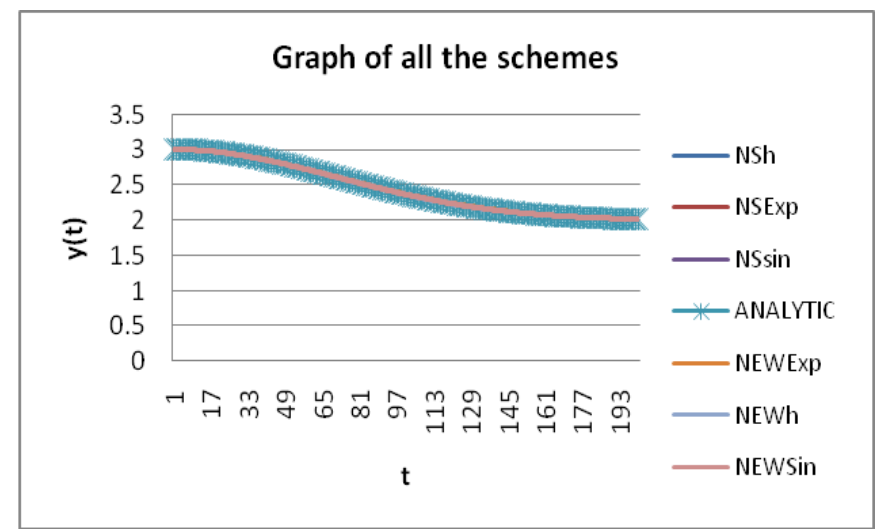

Fig 1: Graph of the schemes of $y^{\prime}=4 x-2 x y, y(0)=3$ 


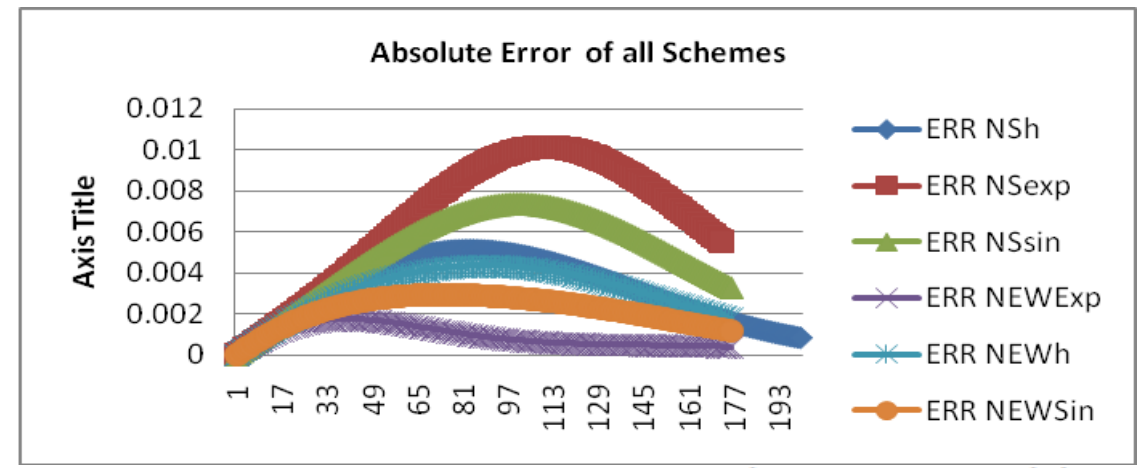

Fig 2: Graph of the absolute error of the schemes of $y^{\prime}=4 x-2 x y, y(0)=3$

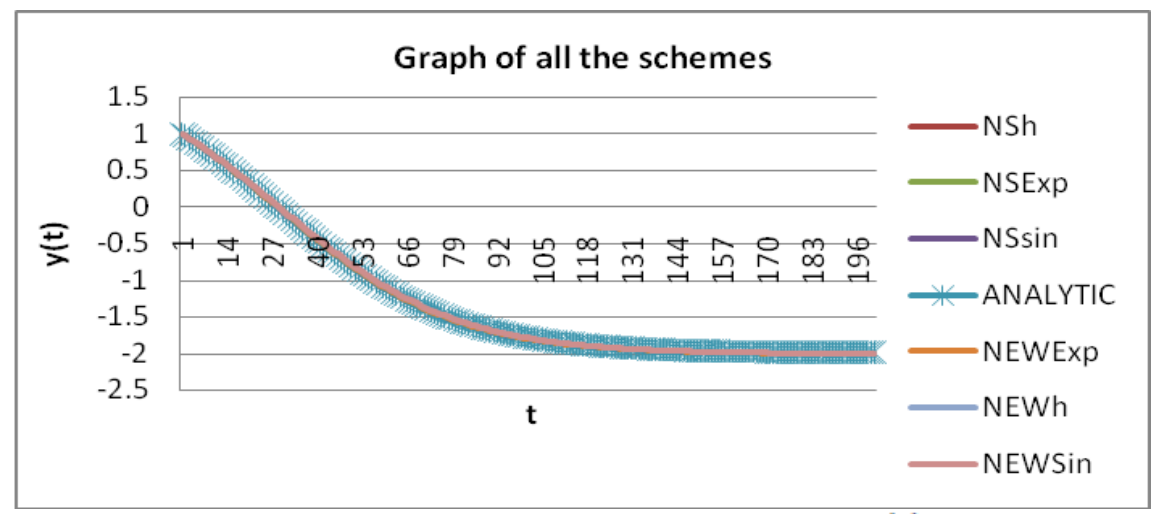

Fig 3: Graph of the schemes of $y^{\prime}=y^{2}-4, y\left(\frac{1}{4}\right)=1$

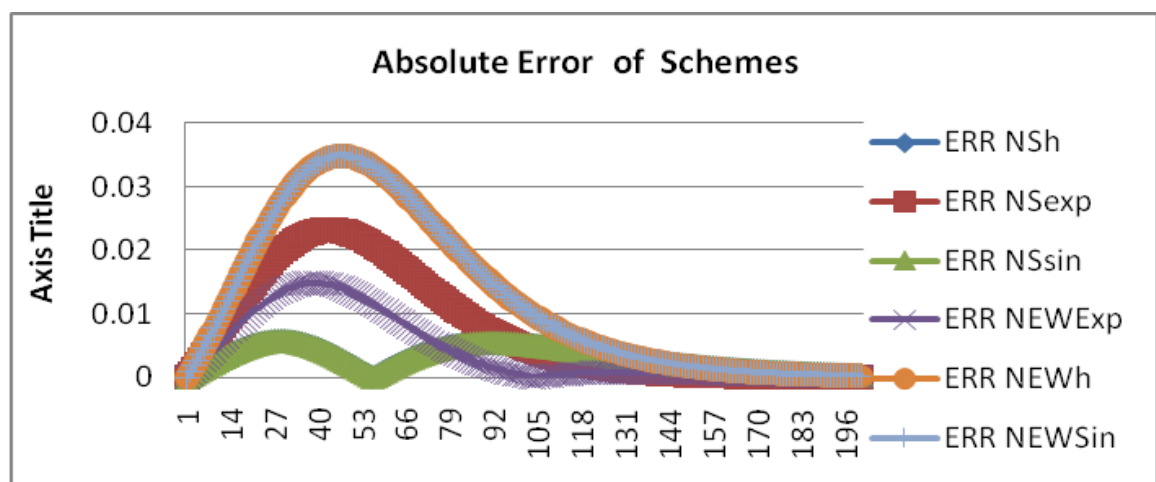

Fig 4: Graph of the absolute error of the schemes of $y^{y}=y^{2}-4, y\left(\frac{1}{4}\right)=1$

\section{Drug Dissemination schemes}

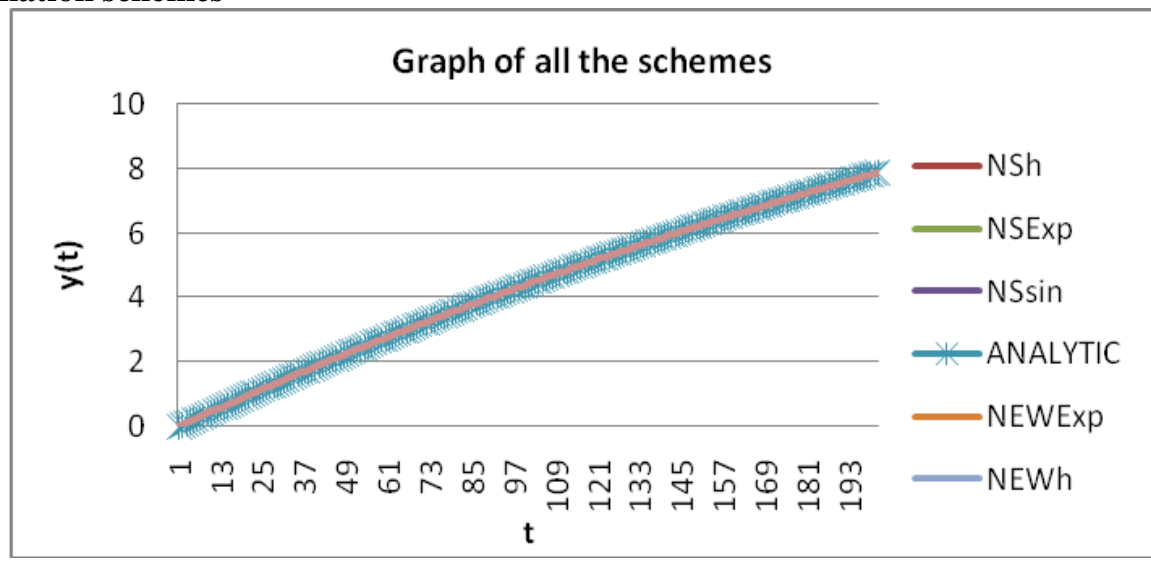

Fig 5: Graph of the schemes of drug dissemination model 


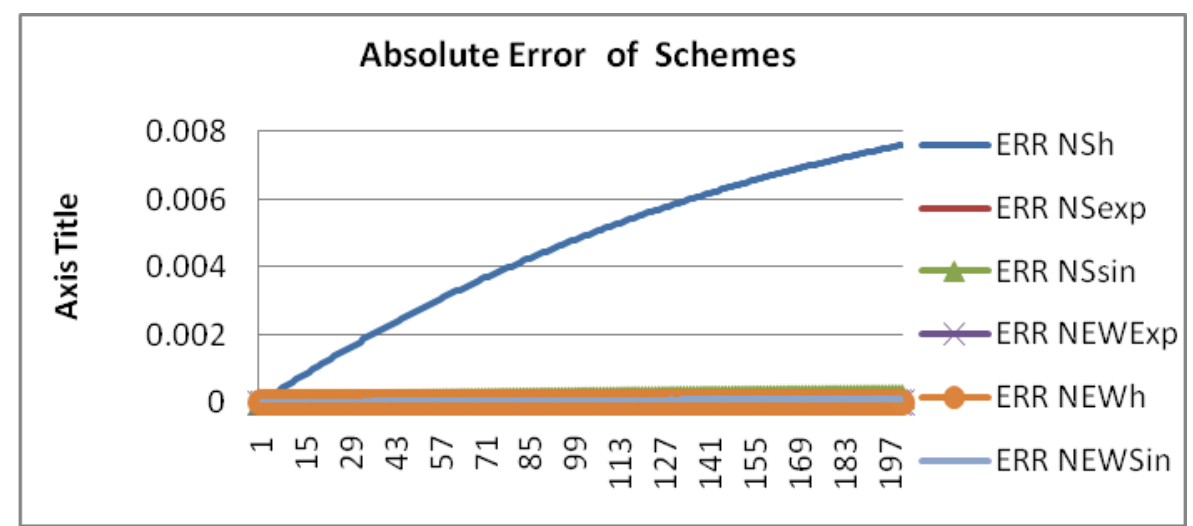

Fig 6: Graph of the absolute error of the schemes of drug dissemination model

\section{Summary and Conclusion}

The curves showed that the schemes have same monotonicity with the analytic solution in all the cases as shown in figures 1, 3 and 5. The use of dynamically calculated step size with our choice of exponential and trigonometric function also produced acceptable results which are sometimes better than using the standard denominator $\mathrm{h}$ as we can see in figure 6 . With carefully selected parameters for the denominator functions, one can be sure of a closer curve to the analytic solution that is stable for all step-size. The renormalization of the standard schemes to obtain the nonstandard schemes is to guarantee that the schemes do not possess numerical instability. The consistency and convergence has been proved analytically. The schemes of the drug dissemination exhibit the lowest form of deviation from the analytic solution as we can see in figure 6 . The deviations are all below $0.1 \mathrm{E}-05$. We can conclude that the proposed discrete model is suitable for the analysis of drug dissolution in human beings as represented by the original differential equation

\section{References}

[1]. Anguelov, R. and Lubuma, J. M. S. (2000). On Mathematical Foundation of the Nonstandard Finite Differential Method, Proceeding of the International Conference on Differential Equations, EQUADIFF' 99 Word Scientific, 1401-1403, Singapore.

[2]. Anguelov, R. and Lubuma, J.M.S (2003). Nonstandard Finite Difference Method by Nonlocal Approximation. Mathematics and Computers in Simulation 6, 465-475.

[3]. Ibijola, E.A., Obayomi A.A and Olabode B. T. (2013). New Nonstandard Finite Difference Schemes for Ordinary Differential Equations whose Solutions can be expressed as Quotient of Two Polynomials, Journal of Emerging Trends in Engineering and Applied Sciences 4(3), 478 - 484.

[4]. Mickens, R. E. (1992). Finite Difference Schemes having the Correct Linear Stability Properties for all Finite Step-sizes. Dynamics Systems and Applications 1, 329-340.

[5]. Mickens, R. E. (1994). Non-standard Finite Difference Models of Differential Equations. World Scientific, 144 - 162, Singapore.

[6]. Mohammed, O. and Hayat, P.A. (2005). Drug Dissemination in Mammals. Adoe Journal of Physical and Biological Sciences, 7, $110-119$.

[7]. Obayomi, A. A. and Oke, M.O. (2015). Development of Nonstandard Discrete Simulation Model for The Numerical Solution Of Second Order Differential Equations. International Journal of Advance Academic Research|Science and Tech. 1(3), 1 - 12 .

[8]. Obayomi, A.A. and Oke, M.O. (2016). Derivation and Implementation of Two-step Finite Difference Schemes for NonAutonomous Second Order Ordinary Differential Equations. Journal of the Nigerian Association of Mathematical Physics, 36, $65-$ 72

[9]. Obayomi, A.A. and Olabode B. T. (2013). A New Family of Non-Standard Schemes for the Logistic Equation. American Journal of Industrial and Scientific Research, 4(3), 277-284, USA.

[10]. Obayomi, A.A., Oke, M.O., Olaosebikan, T.E. and Raji, R.A. (2017). An Hybrid of Numerically Stable Simulation Model for the Solution of Some Initial Value Problems in Ordinary Differential Equations. Global Journal of Mathematics, 9(1), $574-580$.

[11]. Remington Education Publications (2016). Physical Pharmacy, Chapter: Solubility and Dissolution, Pharmaceutical Press

[12]. Zill, D. G. and Cullen, R.M. (2005). Differential Equations with Boundary-Value Problems. Brooks /Cole, Thompson Learning Inc., Canada. 